\title{
NEUROLOGY OF SWALLOWING AND ORAL FEEDING DISORDERS: ASSESSMENT AND MANAGEMENT
}

J Neurol Neurosurg Psychiatry 2003;74(Suppl III):iii48-iii52

\begin{abstract}
$\mathrm{n}$ appreciation of the normal swallowing process ${ }^{1}$ and how it may be affected by neurological disease can inform the clinical diagnosis and management of patients who complain of swallowing problems or present with the nutritional and respiratory complications of failure of oral feeding (table 1).
\end{abstract}

\section{NORMAL SWALLOWING}

Sherrington originally studied swallowing in decerebrate cats. ${ }^{2}$ He described the effect of various agents - whiskey, oil, water-which, when dropped onto the area supplied by the superior laryngeal nerve, would elicit a "swallow". This "swallow" did not involve any preparation of a bolus or any lingual propulsion, and was justifiably considered to be a reflex. I will use the terms reflex or crude swallowing to describe the quintessential components of swallowing, as observed in Sherrington's cat:

- open upper oesophagus

- move bolus into oesophagus

- close airway.

The first two are the sine qua non of swallowing, the third a compulsory accompaniment for oral feeding.

In unconscious humans this sort of swallow is an upper airway protective reflex in response to the unexpected arrival of a bolus in the pharynx. When awake, swallowing is a planned manoeuvre; at a chosen time, with or without a suitably prepared bolus, we swallow. I refer to this as voluntary swallowing, a process initiated voluntarily and not necessarily occurring as a response to stimulation by a bolus.

Bolus preparation is a separate process and may involve chewing (cranial nerve $\mathrm{V}$ motor and sensory), mouth closure (VII), manipulation and retrieval of the bolus (V, VII, XII), and initial propulsion of the bolus (VII and XII).

In the following the term dysphagia is avoided because it can be used to denote symptoms, clinical signs, radiological signs, and as a putative mechanistic explanation for otherwise unexplained nutritional or respiratory problems. Also, instead of the traditional, bolus based separation of swallowing into oral, pharyngeal, and oesophageal phases, a description of the functions involved is used which applies to all swallowing, bolus or no bolus.

So how did Sherrington's cat, sawn off at the mid collicular level, manage to swallow? The following detailed description of crude swallowing may help an understanding of how oral feeding succeeds—or fails—in different neurological diseases.

\section{The crude swallow}

Functionally the larynx and hyoid can be considered as a single structure suspended from the base of the skull by the anterior and posterior suprahyoid musculature ( $\mathrm{V}$ and $\mathrm{Cl}-3$ via the ansa cervicalis, and VII, respectively); the anterior group includes mylohyoid and the anterior belly of digastric, the posterior group stylohyoid, and the posterior belly of digastric. These external laryngeal muscles move the larynx upwards and forwards, a movement easily appreciated by placing two fingers on one's own thyroid cartilage, and then swallowing. The anterior upper oesophageal sphincter, the main muscle of which is the cricopharynx, sits behind the larynx, attached to its posterior aspect. This movement of the larynx pulls the anterior cricopharynx up and forwards, opening the upper oesophagus. Therefore abnormalities of the timing and amplitude of laryngeal movement reduces the duration and extent of upper oesophageal sphincter opening. This leads to pooling, with an increased risk of bolus aspiration caused by simple spill over, or inhalation, from pharynx to larynx. The susceptibility of timing and amplitude of movement to a wide range of neurological conditions suggests that this process may be compromised in many patients. (The swallowing problems after injection of botulinum toxin into the sternomastoids may be caused by toxin weakening the nearby posterior suprahyoid muscles.) 


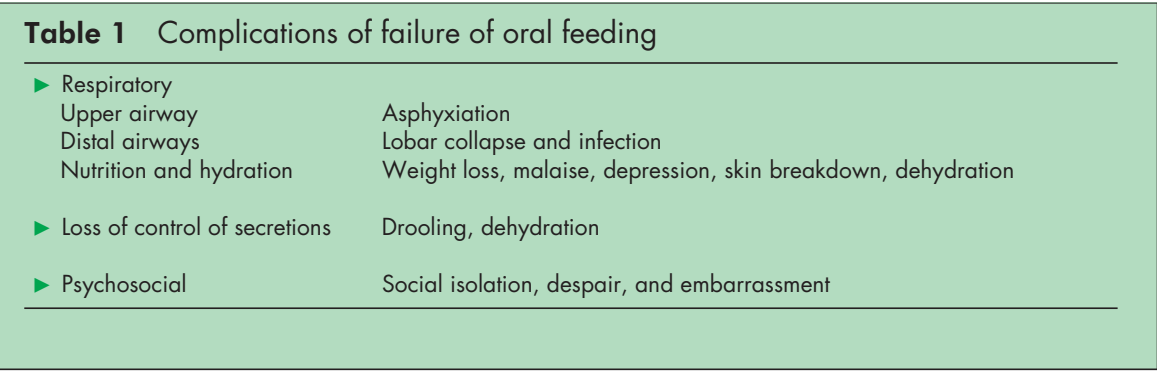

As the sphincter opens the resting pressure falls to zero. The change in pressure exerts a pull on the intra-oral bolus, a phenomenon referred to as hypopharyngeal suction. With the bolus entering the upper oesophagus the subsequent wave of peristalsis produced by apposition of the tongue base and posterior pharyngeal wall acts on the tail of the bolus, clearing the pharynx of any residue.

As the larynx moves up and forwards it exhibits a sphincteric action, and closes; this action is only manifest during swallowing. Closure is at the level of the true vocal cords, the false cords, the arytenoids, and the epiglottis. All of these internal muscles of the larynx are innervated by the Xth cranial nerve, and all but one by its recurrent laryngeal branch.

With the bolus despatched the structures passively return to their starting positions aided by contraction of the infra-hyoid muscles, including sternohyoid and sternothyroid (Cl-3).

Thus the larynx is centre stage in swallowing. If it did not move the upper oesophageal sphincter would not open, and if it did not shut the bolus would always "go down the wrong way".

\section{NORMAL FEEDING}

For swallowing to be able to allow an individual to feed by mouth it has to integrate successfully with other functions, particularly respiration which is necessarily arrested during swallowing. This deglutition apnoea is centrally generated and is synchronous with, but not dependent on, laryngeal closure. Typically exhalation both precedes and follows the swallow, ensuring that bolus remnants in the pharynx are not actively inhaled. Successful feeding also requires effective coughing and intact upper airway reflexes, including retching, gagging, vomiting, and of course crude or reflex swallowing. Their effectiveness is dependent on good respiratory function, and when ineffective they are an important determinant of respiratory complications.

Broadening the view further, there are many potential problems which are common in neurological diseases and which are relevant to feeding. Vigilance, appetite, mood, pain, nausea, dry mouth, ill fitting dentures, availability of informed carers, visual problems (particularly neglect), food consistency, vertigo, and major depression are just some of the determinants of successful feeding in different clinical situations.

Such detailed knowledge about swallowing and feeding is rarely required in routine clinical practice, particularly at the diagnostic stage. However, when considering mechanisms of failure of oral feeding such knowledge can direct discussions with patients and therapists, inform requests for investigations, and prevent inappropriate interventions.

\section{DIAGNOSIS}

The diagnosis of an underlying condition is made using the traditional approach of history, examination, and investiga- tion. An exhaustive discussion of this process is not necessary, but one or two points may merit special mention. While swallowing problems may be the only symptoms volunteered it is usually possible to elicit other relevant symptoms which, when combined with the temporal profile and examination, point the clinician in the right direction. Potentially there are one or two pitfalls if swallowing is the sole problem, as its repertoire of symptoms and signs is so limited and non-specific. Structural diseases, including malignancies in the floor of the mouth, goitres, huge osteophytes, tethering of the larynx after surgery (including tracheostomy insertion), post-irradiation changes in the pharynx and suprahyoid region, and malignancies in the region of the upper oesophageal sphincter may mimic neurological conditions and may not be obvious during routine endoscopy or nasendoscopy. The upper oesophageal sphincter is a region notoriously difficult to visualise in an awake subject, and rigid oesophagoscopy with the patient anaesthetised may be required if there is a strong suspicion of malignancy. Pain on swallowing is an unusual feature of neurological disease, and while there are exceptions (pain in the ascending pharyngeal branches of the external carotid in giant cell arteritis, primary and secondary glossopharyngeal neuralgia) and some curiosities (Eagle's syndrome caused by a prolonged styloid process, pterygoid hamulus bursitis), it is often a feature of malignancy or oesophageal motility problems (nutcracker oesophagus), both of which require careful review by appropriate specialists.

\section{MECHANISMS OF FAILURE OF ORAL FEEDING}

For illustrative purposes diseases with contrasting rates of progression and different mechanisms of swallowing and feeding dysfunction will now be described. I use the term failure of oral feeding to denote nutritional or respiratory complications occurring as a complication of changes in any of the processes involved in feeding, including crude swallowing.

\section{Lateral and medial medullary syndrome}

Infarction involving the medulla may be associated with severe bulbar problems, depending on the cranial nerve nuclei involved.

In lateral medullary syndrome (fig 1) the infarct involves the nucleus ambiguus. These patients have a nuclear 10th nerve palsy. Ipsilaterally there is a palatal and pharyngeal palsy, but more importantly there is a laryngeal palsy. As well as being dysphonic because of the vocal cord palsy, the sphincteric action of the larynx is lost and entry of bolus into the larynx is likely, if not inevitable. This unhinges swallowing, both reflex (crude) and voluntary. Reflex and voluntary coughing is ineffective for the same reason. However, the deficits are not restricted to swallowing. Intra-oral sensory loss, vertigo, nausea, pain, and vomiting all militate against successful oral feeding until significant recovery has occurred. 

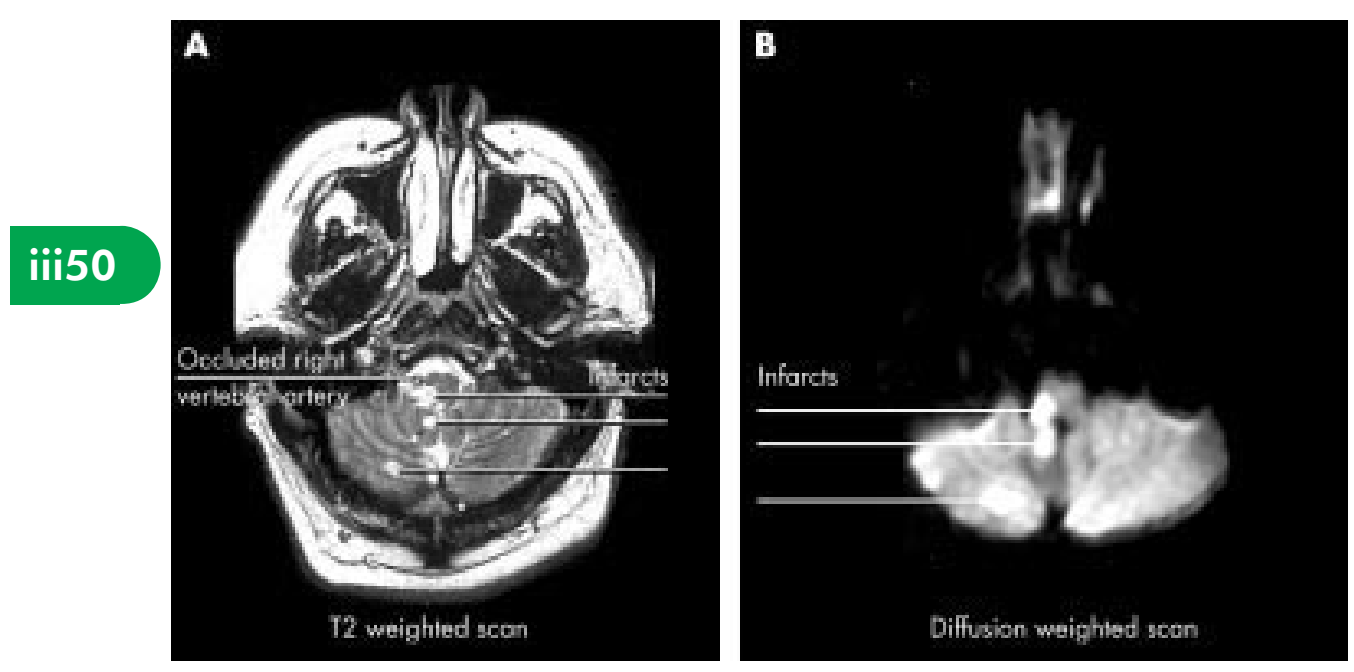

Figure 1 A and B: Lateral medullary (Wallenberg) syndrome-dysphagia; ipsilateral facial sensory loss (pain and temperature); contralateral limb and trunk sensory loss (pain and temperature); ipsilateral arm, leg and gait ataxia; vertigo/vomiting; Horner's syndrome; hiccups. Typically associated with severe swallowing problems caused by the unilateral 10th nerve palsy and the associated dysfunction of the internal musculature of the larynx.

This contrasts with medial medullary syndrome (fig 2) where the unilateral 12th nerve palsy may cause impaired bolus preparation, but preserved laryngeal closure ensures airway protection during reflex and voluntary swallowing. In addition there is less nausea, vertigo, and vomiting and no intraoral sensory loss. These patients are usually able to feed by mouth at an early stage.

In both syndromes the neuronal circuitry of the external laryngeal muscles is essentially intact; the major difference is in the internal laryngeal muscles, unilateral denervation of which compromises all laryngeal functions.

\section{Middle cerebral artery territory stroke}

One of the important features of cerebral hemisphere infarction (fig 3) in relation to bulbar function is the preservation of the lower motor neurone circuitry of the pons and medulla. The larynx, palate, face, pharynx, and tongue are not

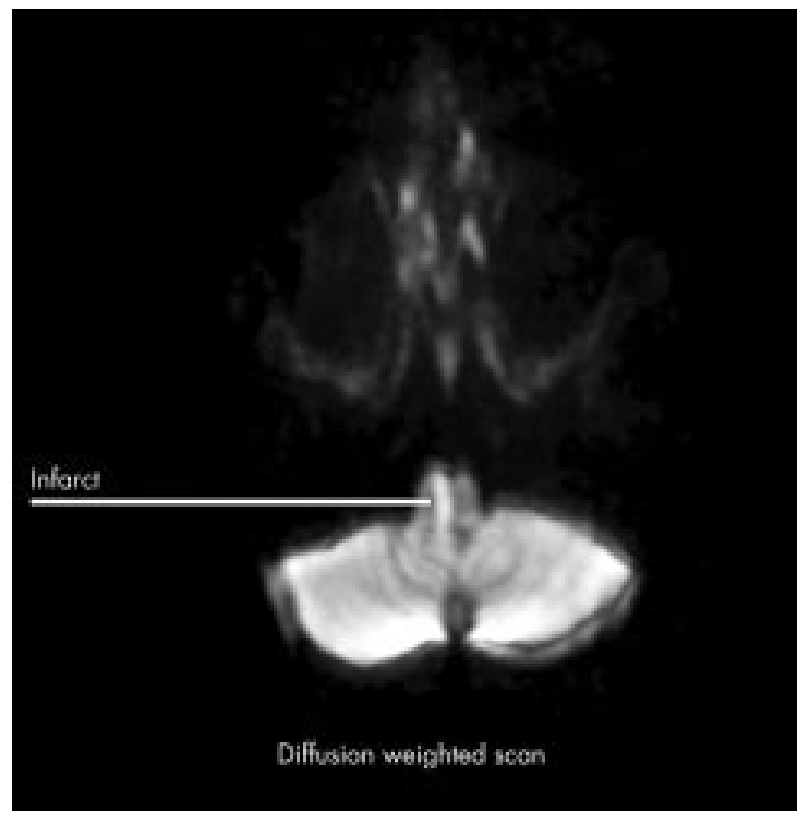

Figure 2 Medial medullary (Dejerine) syndrome-ipsilateral tongue weakness and atrophy; contralateral arm and leg weakness; contralateral limb and trunk sensory loss (vibration and proprioception). Unilateral weakness of the tongue may impair bolus control in the mouth, but laryngeal movement and closure are spared and feeding is possible at an early stage. denervated. What is disrupted is the fine supranuclear control of intra-oral bolus preparation, control, and propulsion.

The clinical situation may be dominated by problems with conscious level, trunk control, limb strength, visual function, vigilance, and cognitive deficits, but the preserved brain stem means that it is theoretically possible for crude swallows to occur and be harnessed to move boluses of suitable consistency from pharynx to oesophagus. Preservation of crude swallowing coupled with reorganisation of supranuclear control $^{3}$ may explain the relatively quick (usually within two weeks) recovery observed. ${ }^{4}$ It may also explain the curious finding, given the complexity of the process as described above, that the loss of pharyngeal sensation has prognostic significance after hemisphere stroke. ${ }^{5}$ This may be because the ability to cooperate successfully with testing of pharyngeal sensation depends on the integrity of the medulla and an adequate level of consciousness, two of the most important components of successful oral feeding. Come back you gag reflex testers, all may be forgiven.

\section{Motor neurone disease (MND/ALS)}

The variable upper and lower motor neurone (UMN and LMN) involvement in motor neurone disease (MND/ALS) means

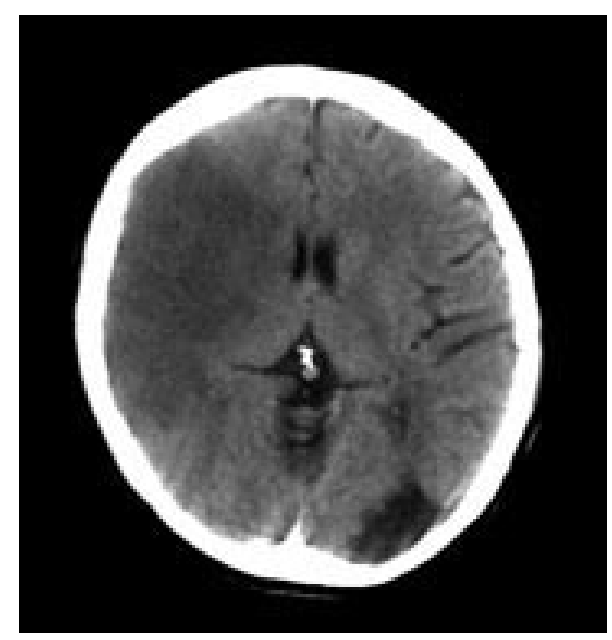

Figure 3 Extensive hemispheric infarction. Failure of oral feeding in this context is caused by a reduction in conscious level. In those who survive, oral feeding may be possible due to a combination of supranuclear reorganisation and the preservation of crude swallowing. 
that the exact mechanism of bulbar dysfunction varies greatly between individuals, and changes with progression of the disease.

In pure upper motor neurone syndromes there is a loss of fine control of the bulbar structures at an early stage causing dysarthria and impaired bolus control. Often patients have exaggerated bulbar reflexes (palatal, pharyngeal, crude swallowing, and masseter), and (crude) swallowing of soft or pureed food is possible even when the patient has no voluntary control of the tongue or face.

An additional factor which allows patients with UMN involvement to continue feeding, despite loss of voluntary control of respiration, is the integrity of the cough reflex; patients may not be able to hold their breath or perform spirometry to command, but in response to laryngeal irritation or aspiration produce very powerful and dramatic coughing.

This reserve of function in UMN bulbar syndromes, which may go unnoticed during the traditional neurological examination, contrasts with lower motor neurone syndromes in which "what you see is what you get". The observed dysfunction in bulbar and respiratory function is the same when used voluntarily by the patient or as part of crude protective responses, and measurements of forced vital capacity are likely to be more representative than in UMN syndromes. This dissociation of voluntary and reflex function makes it difficult to interpret the results of studies using indices of (voluntary) bulbar and respiratory function to select patients for inclusion into trials, and as indices of treatment effect. The patient with predominantly upper motor neurone involvement might not be able to perform a forced vital capacity manoeuvre or do a timed test of swallowing to command, but the preservation of airway protective reflexes, including crude swallowing, may allow them to survive much longer than those who are able to cooperate with testing.

Occasionally the loss of supranuclear inhibition causes troublesome gagging - for example, during teeth cleaning and feeding. The glottopharyngeal reflex, in health, makes the vocal cords partly adduct in response to stimulation in the pharynx, as if in readiness for laryngeal closure. An exaggerated version may be responsible for some of the dramatic coughing and choking episodes seen in MND patients in response to apparently trivial stimuli.

\section{Multisystem atrophy}

The backdrop of parkinsonism will have predictable effects on the speed and amplitude of movement of the face, jaw, and tongue. Reduced hyolaryngeal elevation will affect the opening of the upper oesophageal sphincter. If anticholinergic drugs are used the resultant dry mouth may be troublesome and oesophageal motility may be adversely sensitive to anticholinergic medication in parkinsonian syndromes. In addition to this patients may develop oromandibular and cervical dystonias, including a disproportionate antecollis, each of which further compromises bolus control.

If laryngeal dysfunction is a prominent feature (Shy Drager syndrome) it can be highly selective, at least initially, for the abductors of the vocal cords. However, the sphincteric action of the larynx during swallowing is relatively preserved. For this reason multisystem atrophy patients may require a tracheostomy, to secure the airway, but feed successfully by mouth. The insertion of a tracheostomy may reduce hyolaryngeal movement further, but in practice this does not appear to be a problem.

\section{Oculopharyngeal muscular dystrophy}

This condition is characterised by profound and selective weakness without any central or peripheral cranial nerve involvement. The preservation of upper motor neurone control means that, strength permitting, the bolus can be prepared with reasonable precision and initiation of bolus propulsion is under voluntary control.

As a group these patients have, in the past, tended to be treated by cricopharyngeal myotomy. The evidence for this intervention having a beneficial effect is unconvincing. ${ }^{6}$ This may be because the radiological observation of supposed "spasm" may be due to weak suprahyoid muscles failing to pull the sphincter open rather than there being true spasm itself. It is counterintuitive to think of a muscle being in "spasm" in a condition characterised by flaccidity and weakness. The same reservations may be expressed about cricopharyngeal myotomy in MND/ALS where the feeding problem is the result of many abnormalities, not just those causing impaired opening of the upper oesophageal sphincter.

\section{MANAGEMENT}

In the absence of prospective randomised trials a pragmatic approach is required. It is acknowledged in stroke that there is a dearth of randomised trials looking at the management of swallowing and feeding problems. ${ }^{7}$ In slowly progressive neurological diseases, significant weight loss, chest infections or the development of slow, unenjoyable, and effortful oral feeding are indications for adjunctive feeding. In practice such clinical features seem to have a greater validity than any surrogate measures. Studies in stroke suggest that gastrostomy insertion provides the easiest and most effective feeding route. ${ }^{8}$ The timing of insertion in patients with a progressive disease is difficult. It may be interpreted by the patient only as a sign of the end stages of the disease rather than a device which may enhance quality of life. If the patient faces inevitable bulbar failure and accompanying deficits, particularly respiratory, earlier rather than later PEG (percutaneous endoscopic gastrostomy) insertion must be preferable, providing that this is compatible with the person's wishes. Patients may find that a limited oral intake remains enjoyable even when they do not depend on it to maintain nutrition.

Following acute events, such as stroke, the clinical approach has to be more tentative, and an appreciation of the multifactorial nature of failure of oral feeding suggests that single surrogate clinical or radiological indices are unlikely to be useful predictors of feeding ability in different patient groups with different stroke syndromes. Videofluoroscopy is considered to be a gold standard in the assessment of swallowing dysfunction. ${ }^{9}$ The anatomical detail and the immediacy of the sight of the bolus in transit produces an arresting image. Abnormalities vividly demonstrated by videofluoroscopy include:

- premature escape of the bolus from the mouth

- impaired oral control

- impaired opening of the upper oesophageal sphincter

- aspiration into the larynx.

Less clear is whether or not the images, and the large amount of data that can be extracted from them, provide the answers to important clinical questions regarding the appropriateness and safety of oral feeding. In most guidelines the presence of an adequate conscious level and the ability to sit up is taken as a sign that patients are eligible to commence trials of oral feeding with small amounts of an appropriate consistency. Their feeding ability is then established over a representative time period using foods of an appropriate texture introduced in a controlled manner. 
This issue seems to illustrate a subtle but profound difference between diseases and abilities. As clinicians we can deduce through a reductionist approach the pathological diagnosis. However we are not able to use the same information to predict accurately an ability, whether it be the ability to swallow a pureed diet safely, drive a motor car in heavy traffic, or play the bassoon to grade VIII standard. In those judged to be eligible, abilities can be tested but not diagnosed.

\section{Oral secretions}

Transdermal preparations of anticholinergic drugs or subcutaneous administration of glycopyrronium bromide are effective in some patients with troublesome drooling. Botulinum toxin, injected into the parotid glands, offers a convenient route of administration without risk of systemic anticholinergic side effects, but reports of its use are limited and anecdotal.

In highly selected patients surgical interventions may be considered for intractable aspiration. ${ }^{10}$ Options include surgical manipulation of the arytenoids, partial or complete closure of the laryngeal entrance, and laryngectomy with an end tracheostome. Obviously phonation is permanently compromised or lost depending on the extent of surgery. An alternative is sectioning of the larynx below the true vocal cords, leaving the option of reconnecting the larynx and trachea if subsequent recovery of the intrinsic laryngeal muscles occurs.
Personal experience ${ }^{11}$ suggests that the individual patients must be highly motivated, be well enough to undergo major surgery, have agreed on a clearly identified goal of intervention, and to have failed to respond to more conservative approaches.

\section{ACKNOWLEDGEMENT}

I would like to acknowledge the help and support of Professor Mark Wiles.

\section{REFERENCES}

1 Miller AJ. Deglutition. Physiol Rev 1982;62:129-84.

2 Miller FR, Sherrington CS. Some observations on the buccopharyngeal stage of reflex deglutition in the cat. Q J Exp Physiol 1916;9:147-86.

3 Hamdy S, Aziz Q, Rothwell JC, et al. Explaining oropharyngeal dysphagia after unilateral hemispheric stroke. Lancet 1997;350:686-92.

4 Gordon C, Langton Hewer R, Wade DT, et al. Dysphagia in acute stroke. BM 1987;295:411-4

5 Kidd D, Lawson J, Nesbitt R, et al. Aspiration in acute stroke: a clinical study with videofluoroscopy. QJM 1993;86:825-9.

6 Bucholz DW. Cricopharyngeal myotomy may be effective treatment for selected patients with neurogenic oropharyngeal dysphagia. Dysphagia $1995 ; 10: 255-8$

7 Bath PM, Bath FJ, Smithard DG. Interventions for dysphagia in acute stroke. Cochrane Database Systemic Review. 2000;(2):CD000323.

8 Norton B, Homer-Ward M, Donnelly MT, et al. A randomised prospective comparison of percutaneous endoscopic gastrostomy and nasogastric tube feeding after acute dysphagic stroke. BM 1996;312:13-16.

9 Logemann JA. Evaluation and treatment of swallowing disorders. San Diego: College Hill, 1983.

10 Baredes S. Surgical management of swallowing disorders. Otolaryngol Clin North Am 1988;21:711-20.

11 Hughes TAT, Wiles CM. Neurogenic dysphagia: the role of the neurologist. J'Neurol Neurosurg Psychiatry 1998;64:569-72.

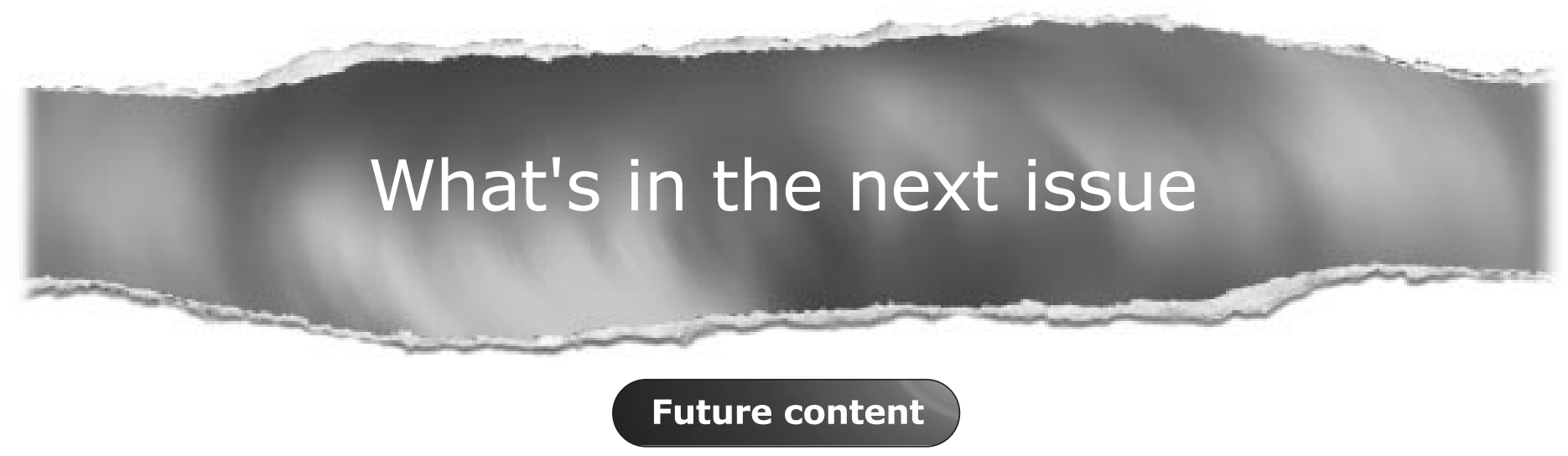

See which articles have just been accepted for publication and preview the table of contents for the next issue a month before it is published

www.jnnp.com 